\title{
Evaluation of the Level of CXCL10 in Patients with Parainfluenza Virus Type III (HPIV3)
}

\author{
Noor Hameed Alkharsan ${ }^{1}$, Siham Jasim Al-kaabi ${ }^{1}$ \\ ${ }^{1}$ Department of biology, Faculty of Education for women, University of Kufa, Najaf, Iraq;
}

\begin{abstract}
Acute respiratory illness could be caused by Human parainfluenza viruses (PIVs) in children, the old, and immunocompromised patients. The frequent and common cause of pneumonia and bronchiolitis is PIV3, whilst the upper respiratory tract illness and croup are frequently caused by PIV1 and PIV2. However, this study aimed to highlight a group of patients with type III influenza virus. 29 sample took from people suffering from upper and lower respiratory diseases as well as 27 conrol sample to assessing the level of a type of cellular motility in their catheter, cimclin cxcl10 by the use of ELISA. The study also showed that there were no significant differences between Male Injury Rate Now as well as an inverse relationship between the level of cimokin cxcl10 and age. Moreovere, this study showed an increase in the level of cimcin cxcl10 in the case of HPIV infection compared with the control group.
\end{abstract}

Keywords: CXCL10; HPIV3; Respiratory infection

\section{Introduction}

Human parainfluenza viruses (HPIVs) are among the most common cause of acute airway illness in children, the elderly and immunocompromised patients ${ }^{(1)}$ the elderly, and immunocompromised patients. PIV3 is a common cause of bronchiolitis and pneumonia, whereas PIV1 and 2 are frequent causes of upper respiratory tract illness and croup. To assess how PIV1, 2, and 3 differ with regard to replication and induction of type I interferons, interleukin-6, and relevant chemokines, we infected primary human airway epithelium (HAE. HPIV consists of four major serotypes, PIV4 is a common respiratory pathogen which is alike to PIV3 in clinical demonstration ${ }^{(2)}$, whilst the upper respiratory tract illness and croup are frequently caused by PIV1 and PIV2 ${ }^{(1)}$ the elderly, and immunocompromised patients. PIV3 is a common cause of bronchiolitis and pneumonia, whereas PIV1 and 2 are frequent causes of upper respiratory tract illness and croup. To assess how PIV1, 2, and 3 differ with regard to replication and induction of type I interferons, interleukin-6, and relevant chemokines, we infected primary human airway epithelium (HAE. Due

\section{Corresponding author:}

Noor Hameed Alkharsan

E-Mail address: noorh.alkhrsain@gmail.com to optimal viral replication inside epithelial cells of the respiratory tract.HPIV3 is the common frequent in the worldwide amongst the four familiar serotypes that cause of hospitalization respiratory illness. Notwithstanding this high difficulty of illness, there is not any authorized vaccine or therapy to handle or even limiting this high difficulty of illness ${ }^{(3)}$.

HPIV-3 is a virus which belongs to the Paramyxoviridae group, non-segmented, an enveloped as well as negative sense RNA virus ${ }^{(4)}$ non-segmented, negative sense RNA virus that belongs to the Paramyxoviridae family. HPIV-3 is a common cause of bronchiolitis and pneumoniae in children less than 1 year of age and one of the leading causes of acute lower respiratory tract infections in children under five years of age. In Israel, the epidemiology of HPIV-3 infections is not well characterized. In this study, epidemiology and molecular characterization of HPIV-3 was performed on patient samples collected between January 2012 and September 2015. Nasopharyngeal swabs ( $N=$ 15,946. HPIV-3 infecting lower airway epithelial cells induces bronchiolitis and pneumonia in children less than one year of age and one of age and it is considered as the leading cause of acute lower respiratory tract infections in children under five years of age ${ }^{(4)}$ nonsegmented, negative sense RNA virus that belongs to the Paramyxoviridae family. HPIV-3 is a common cause 
of bronchiolitis and pneumoniae in children less than 1 year of age and one of the leading causes of acute lower respiratory tract infections in children under five years of age. In Israel, the epidemiology of HPIV-3 infections is not well characterized. In this study, epidemiology and molecular characterization of HPIV-3 was performed on patient samples collected between January 2012 and September 2015. Nasopharyngeal swabs ( $N=15,946 .{ }^{(5)}$ reported that HPIV3 may lead to asthma exacerbations in children and adults. Respiratory viruses contributing to the pathophysiology of lower and upper respiratory disorders by invading the epithelium activate innate immune response and induce inflammatory cytokines release. However, the effects of HPIV3 infection have not been well-defined on nasal epithelial cells (HNECs).

Chemokines are small, structurally related proteins, one of them is the chemokine interferon- $\gamma$ (IFN- $\gamma$ ) inducible protein CXCL10 and also called IP-10 is a $10 \mathrm{kDa}$ protein, which is functionally categorized as a T helper 1- (Th1) chemokine. Furthermore, CXCL $\square 10$ is a member of the CXC chemokine which is the ligand of CXCR3, and regulates immune responses through the activation and recruitment of leukocytes, such as, $\mathrm{T}$ cells, eosinophils, and monocytes to induce chemotaxis, apoptosis, cell growth and angiostasis (6).

Alterations in CXCL10 expression levels have been associated with inflammatory diseases such as immune dysfunction, infectious diseases, and tumor development. According to (7), the severity of various diseases can be predicted as well as recognized through CXCL10. Th1 orientated immune response is the special case of the host immune response that can be determined through CXCL10 level in blood. According to (8), CXCL10 is stimulated through Tumor necrosis factor- $\alpha$ and IFN- $\gamma$ which is produced by Th1 lymphocytes in inflamed tissues recruited. The common abundant chemokines induced are The $\mathrm{T}$ cell chemoattractants CXCL11 and CXCL10. Differences in replication and cytokine secretion might explain some of the differences in PIV epidemiology and serotype-specific pathogenesis(1)the elderly, and immunocompromised patients. PIV3 is a common cause of bronchiolitis and pneumonia, whereas PIV1 and 2 are frequent causes of upper respiratory tract illness and croup. To assess how PIV1, 2, and 3 differ with regard to replication and induction of type I interferons, interleukin-6, and relevant chemokines, we infected primary human airway epithelium (HAE. In order to adequately recognize determinants of variability of immune responses to respiratory virus infections of airway epithelial cells in patients, this study focused mainly on understanding of the role of CXCL10 in HPIV3 infected patients.

\section{Materials and Method}

\section{Blood samples}

Blood samples were collected from people suffering from upper and lower respiratory diseases. The samples were collected from Al-Sadr Teaching Hospital and AlHakim General Hospital in Najaf Governorate. $5 \mathrm{~mL}$ of venous blood was withdrawn using a sterile syringe placed in a test tube containing no anticoagulant. The blood was then left at room temperature for half an hour; the serum was then removed from the rest of the blood components using a sterile microbial. It was distributed in five Pendrove tubes and 200 microliters per tube for the purpose of avoiding melting. , And was frozen (20m) until the test (9).

\section{Method}

The antibodies against Paranfluenza (IgM) was determined in patients' and control urine, using ELISA technique, the test was performed according to the method reported and supplied with the VIRCELL test kit.

The number of tests used by Ray Biotech was used to measure the quantitative level of the public health laboratory in Najaf Governorate, as follows: The doublecheck factor and the human control factor (CXCL10) are studied in serum samples and by the associated immunosorbent system (Sandwich ELISA)

\section{Statistical Analysis}

The data were analyzed statistically using computerized SPSS v.24. Non- parametric tests has been used for variables that were n't followed the normal distribution such as Kruskal-Wallis Test (Multiple Comparisons) and Mann-Whitney Test, and Chi-square Test. ANOVA has been employed for variables that followed the normal distribution. Also, Microsoft Excel 2016 has been adopted to build histograms and figures of the correlation test. Data were expressed as (mean $\pm \mathrm{SE}$ ), statistically significant at $\mathrm{p}$-value 0.05 .

\section{Result and Discussion}

Many studies have focused on HPIV-3 infection in children because of high positive rate and morbidity of 
HPIV infection in kids ${ }^{(10)}$, therefore less is known about HPIV2 and HPIV-3 infection in adults.

In this study, 88 blood samples were collected from hospitalized HPIV-3 patient suffered from upper and lower respiratory airway after clinical diagnosis by the specialist doctor. The samples were collected from AlSadr Teaching Hospital and Al-Hakim General Hospital in Najaf Governorate, compared to 27 healthy people who did not suffer from respiratory or other chronic diseases, they were considered a control group.

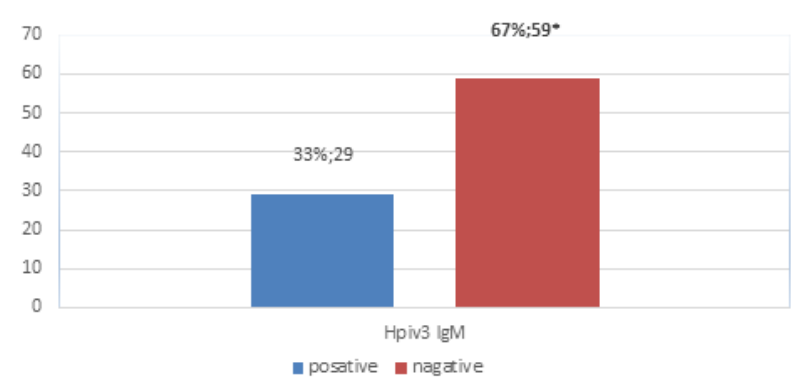

Figure (1) Spread of Parainfluenza virus type III HPIV3 in patient samples

The study demonstrated that the majority of HPIV3 patients had chronic infection negative IgM 59 (67\%) while, 29 (33\%) of HPIV3 patients had acute infection (positive IgM) as depicted in figure(1).This result somewhat compatible with previous studies have reported that, the majority of antibody levels of specific immunoglobulin $\mathrm{G}$ ( $\mathrm{IgG}$ )-class antibodies in serum samples collected during the convalescent phase of the disease over the detection of specific IgM present during the acute phase of infection. ${ }^{(11)}$

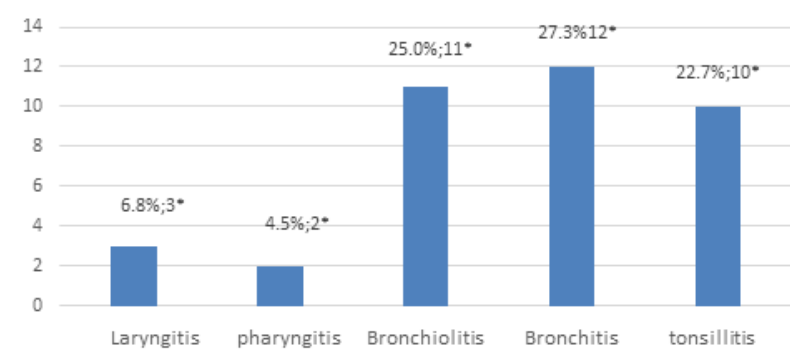

Figure (2): Percentage of clinical signs of patients infected with parainfluenza hpiv3

In figure (2) HPIV3 infections cause significant morbidity and burden of hospitalization. Clinical diagnosis revealed, $27.3 \%$ had bronchitis, bronchiolitis $25 \%$, tonsillitis $22.7 \%$, laryngitis $6.8 \%$, and $2.4 \%$ had pharyngitis, these finding agree with the study noted that laryngeal trachea bronchitis (LTB) was most often caused by HPIV 1 and HPIV 2 with only a single case caused by PIV 3, cases of bronchiolitis occurred during infection were $26 \%$, while $70 \%$ cases of pneumonia were caused by PIV $3^{(12)}$.

PIV in children accounted for $6.8 \%$ of all hospitalizations for fever and/or acute respiratory illnesses. HPIV3 is the most frequent cause of hospitalization, followed by HPIV1 and HPIV2 (13). Some study reported that, the most common presenting symptom was cough $78 \%$ but other signs and symptoms of acute respiratory tract infection were common. $62 \%$ of patients had a documented fever upon presentation, and $40 \%$ of patients presented with gastrointestinal complaints of vomiting, diarrhea ${ }^{(12)}$, and our findings agree with the previous clinical reports that, almost all patients had some respiratory symptoms, the majority had cough $63.6 \%$, and a large proportion also had documented breathlessness was $59.1 \%$, fever $54.5 \%$, vomiting $29.5 \%$ and rhinorrhea was $25 \%$, Both of clinical diagnosis and symptoms have significant differences at $\mathrm{P}<0.05$ figure (3).

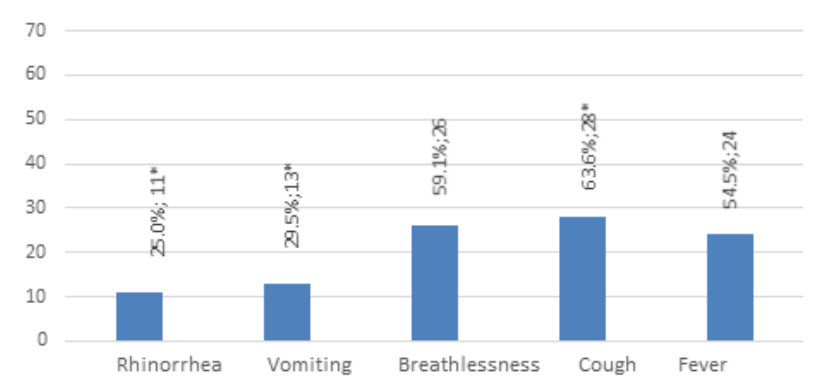

Figure (3): Percentage of clinical symptoms of patients with HPV3

HPIVs are common community acquired respiratory pathogens responsible for respiratory infections throughout the world without any gender, ethnic, age, or geographic boundaries ${ }^{(10)}$; however, morbidity and mortality rates are higher in patients living in poor countries such as rural areas without access to supportive care as compared to developed countries ${ }^{(14)}$.

This study shown that HPIVs were more commonly isolated from females patients than male in patient with acute infection (positive $\operatorname{IgM}$ ), they were 10 males and 19 females, their ages ranged from 20 to more than 50 years with (Mean \pm SD) $(39.97 \pm 2.83)$, a control group with mean age $40.85 \pm 2.79$, the Patients from cities were 14 and rural areas were 15 Patients. Therefore, no significant differences were found in the result of age, sex and 
housing between patient and control group. While other studies founded that, the median age (77.5 years; range, 19.0 - 84.0 years) of the 24 HPIV-3-infected patients was higher than the median age (63.0 years; range, 18.0 99.0 years) of 961 patients who tested HPIV-3-negative and also agree with us no significant differences found (P 5. 04) ${ }^{(15)}$ we tested acute- and convalescent-phase serum specimens from hospitalized adults participating in a population-based prospective study of lower respiratory tract infection during 1991-1992. We tested all available specimens from the epidemic seasons for each virus and $\sim 300$ randomly selected specimens from the corresponding off-seasons for antibodies to HPIV1, HPIV-2, or HPIV-3. During the respective epidemic season, HPIV-1 infection was detected in 18 (2.5 $\mid \backslash \backslash \%$, which was older than our findings it can be because the result of the past decades.

PIV infectio $n$ causes a spectrum of diseases associated with the expression and release of proinflammatory mediators, PIV-infected patients had higher nasal wash concentrations of chemokines such as CXCL8, CXCL9 and CXCL10 as compared to uninfected control patients ${ }^{(12)}$. A specific diagnosis of a lower and upper respiratory viral infection is often difficult despite frequent clinical suspicion, may be improved the diagnostic tools by use of sensitive detection methods and biomarkers. The previous study demonstrated, that the $\mathrm{T}$ cell chemo attractants CXCL10 one of the most abundant chemokines induced in HPIV3 ${ }^{(1)}$ the elderly, and immunocompromised patients. PIV3 is a common cause of bronchiolitis and pneumonia, whereas PIV1 and 2 are frequent causes of upper respiratory tract illness and croup. To assess how PIV1, 2, and 3 differ with regard to replication and induction of type I interferons, interleukin-6, and relevant chemokines, we infected primary human airway epithelium (HAE. Human airway epithelial cells may generate CXCL10 in response to virus infection with high concentrations. In particular, have been correlated with more severe HPIV disease ${ }^{(16)}$.

This study findings support previous research, where this study found a significant difference in CXCL10 concentrations in patients when compared to control cases $(\mathrm{P}<0.001)$, whereas CXCL10 concentrations levels were higher in patients than control group, the mean was $2023.98 \pm 273.13$ and $776.62 \pm 86.79$, respectively. Moreover other study suggested that, respiratory viruses can be found in patients with serious acute airway illness CXCL10 may be a useful biomarker for viral ARI (as opposed to non-viral) by use of PCR assays more frequently than previously appreciated (17) clinical predictors and inflammatory mediator profile of respiratory viral infection in serious acute respiratory illness were investigated. Sequential bronchoalveolar lavage (BAL.

A study conducted by ${ }^{(18)}$ on 164 healthy subjects with age ranges from 10 to 79 year-old. They reported that serum levels of CXCL10 and SCCL2 were significantly increased in older individuals $(\mathrm{r}=0.32, \mathrm{P}$ $<0.001$ ). This finding is in contrary with our finding, where, a significant negative correlation between was found between the serum level of CXCL10 and older HPIV3 patients ( $\mathrm{r}=-0.428, \mathrm{P}=0.020)$. This might be due to the small number of patients examined in the current study as compared to that of their study.

To compare the result associated with this study, we classified HPIV3 patient with acute infection (positive IgM group) into three different age groups. Group I consisted of 13 patients with age range from 20-34, group II consisted of 6 patients with age range 35-49 and group III 10 patients with age more than 50 year and were each group appears normally distributed using descriptive statistics.

Table (1): The effect of age in the concentration of CXCL10 among the group of Hpiv patients (Hpiv3 IgM) compared to control group

\begin{tabular}{|l|l|l|l|}
\hline Age group & Control (SD \pm mean) & CXCL10 (SD \pm mean) & Number of patients \\
\hline $20-34$ & $966.62 \pm 145.07$ & $2304.42 \pm 435.4$ & 13 \\
\hline $35-49$ & $966.62 \pm 145.07$ & $2148.63 \pm 820.91$ & 6 \\
\hline$\geq 50$ & $696.52 \pm 110.22$ & $1584.62 \pm 293.29$ & 10 \\
\hline
\end{tabular}


In Table (1), figure (4) A significant relationship between CXCL10 among different age group of HPIV3 patients compared to control group was founded in this study with $\mathrm{P}<0.05$, the concentration of CXCL10 for the age group I was $2304.42 \pm 435$ compared to control group $(145.07 \pm 966.62) \mathrm{pg} / \mathrm{ml}$, whereas the concentration of CXCL10 for the age group II was $2148.63 \pm 820.91$ compared to the control group (145.07 \pm 966.62$) \mathrm{pg} / \mathrm{ml}$. In the group III CXCL10 concentration $1584.62 \pm 293.29$ was compared with control group (110.22 \pm 696.52$) \mathrm{pg} / \mathrm{m}$. This is agreeing with the study which mentioned that, decrease in pro-inflammatory cytokine expression by aging in bronchoalveolar lavage patients ${ }^{(19)}$ the natural variation of cytokine expression in healthy horses has yet to be described. The objectives of this study were to: (1. This finding is in contrary with ${ }^{(20)}$ study, increase of pro-inflammatory cytokines production by age-associated may be a co-factor for the pathogenesis of airway diseases, where pro-inflammatory cytokines in humans such as CXCL10 are increase with age, the phenomenon known as 'inflamm-aging'.

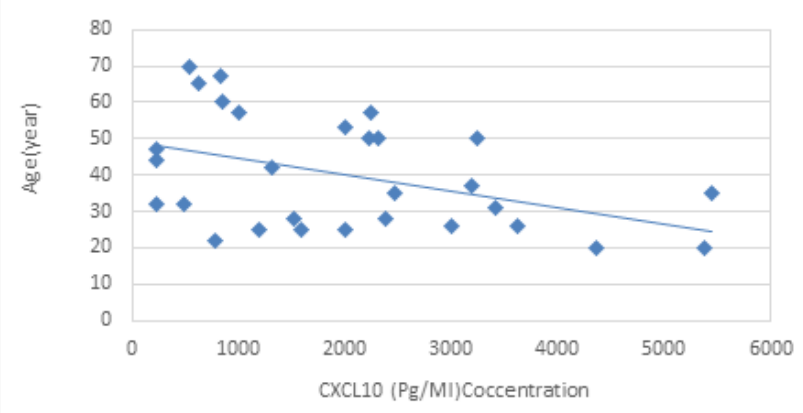

Figure (4):An inverse (negative) relationship between CXCL10 concentration and age in patients with HPV3 ( $r=$ $-0.428 *, p=0.020$

Table (2) the effect of sex in the concentration of CXCL10 among the group of HPIV patients (Hpiv3 IgM) compared to control group

\begin{tabular}{|l|l|l|l|}
\hline $\begin{array}{l}\text { Group of } \\
\text { study }\end{array}$ & Gender & SD \pm mean & Total \\
\hline \multirow{2}{*}{ Patient } & Male & $\begin{array}{l}* 385.72 \pm \\
2001.66\end{array}$ & \multirow{2}{*}{29} \\
\cline { 2 - 4 } & Female & $\begin{array}{l}* 327.73 \pm \\
2066.38\end{array}$ & \\
\hline \multirow{2}{*}{ Control } & Male & $134.50 \pm 771.04$ & \multirow{2}{*}{27} \\
\cline { 2 - 4 } & Female & $116.38 \pm 781.81$ & \\
\hline
\end{tabular}

On the other hand, according to gender in table (2), the observed association of HPIV3 with over expression of CXCL10 were more pronounced in female than males in both patient and control group, the concentration of CXCL10 for males patients was $(2001.66 \pm 385.72 *)$ pg / $\mathrm{ml}$ compared with the control group $(771.04 \pm 134.50)$ pg / ml, while the CXCL10 concentration for females patients was $(2066.38 \pm 327.73 *) \mathrm{pg} / \mathrm{ml}$ compared to the control group $(781.81 \pm 116.38) \mathrm{pg} / \mathrm{ml}$. This finding is in agreement with the result of (18) reported that CXCL10 was slightly higher in healthy females than that of males. This may be due to estrogen enhanced CXCL10 expression (21). But this study finding is contrary what mentioned before, over expression of CXCL10 were more pronounced in males than in female patients with Cerebral malaria (22)CXCL10, is a strong predictor of both human and experimental cerebral malaria. Increased plasma and cerebrospinal fluid levels of CXCL10 were tightly associated with fatal CM in Indian and Ghanaian patients. In the present study, we hypothesized that in a subset of malaria patients, CM susceptibility is associated with variation in CXCL10 expression. We determined whether polymorphisms in the CXCL10 gene promoter region played a role in the clinical status of malaria patients and addressed the genetic basis of CXCL10 expression during malaria infection. Following extensive bioinformatics analyses, two reported single nucleotide polymorphisms in the CXCL10 promoter $(-135 \mathrm{G}>\mathrm{A}$ [rs56061981] and $-1447 \mathrm{~A}>\mathrm{G}$ [rs4508917]. Further study involving larger sample size is recommended for evaluating usefulness of these markers in HPIV3 patients.

Ethical Clearance- Obtained from University of Kufa from Faculty of Education for women, Department of biology.

\section{Source of Funding- Self \\ Conflict of Interest - Nil \\ References}

1. Schaap-Nutt A, Liesman R, Bartlett EJ, Scull MA, Collins PL, Pickles RJ, et al. Human parainfluenza virus serotypes differ in their kinetics of replication and cytokine secretion in human tracheobronchial airway epithelium. Virology [Internet]. 2012;433(2):320-8. Available from: http://www.sciencedirect.com/science/article/pii/ S0042682212004084

2. Frost HM, Robinson CC, Dominguez SR. 
Epidemiology and clinical presentation of parainfluenza type 4 in children: a 3-year comparative study to parainfluenza types 1--3. J Infect Dis. 2013;209(5):695-702.

3. Faure M. The p value of HPIV3-mediated autophagy inhibition. Cell Host Microbe. 2014;15(5):519-21.

4. Jornist I, Mendelson E, Ram D, Azar R, Mandelboim M, Hindiyeh M. Molecular characterization of human parainfluenza virus type 3 (HPIV-3) among hospitalized patients from central Israel. J Clin Virol. 2016;82:S112.

5. Lewandowska-Polak A, Brauncajs M, Paradowska E, Jarzlkebska M, Kurowski M, Moskwa S, et al. Human parainfluenza virus type 3 (HPIV3) induces production of IFN $\$ \gamma \$$ and RANTES in human nasal epithelial cells (HNECs). J Inflamm [Internet]. 2015 Feb;12(1):16. Available from: https://doi. org/10.1186/s12950-015-0054-7

6. Lee EY, Lee Z-H, Song YW. CXCL10 and autoimmune diseases. Autoimmun Rev [Internet]. 2009;8(5):379-83. Available from: http:// www.sciencedirect.com/science/article/pii/ S1568997208002437

7. Liu M, Guo S, Hibbert JM, Jain V, Singh N, Wilson NO, et al. CXCL10/IP-10 in infectious diseases pathogenesis and potential therapeutic implications. Cytokine Growth Factor Rev [Internet]. 2011;22(3):121-30. Available from: http://www.sciencedirect.com/science/article/pii/ S1359610111000293

8. Antonelli A, Ferrari SM, Giuggioli D, Ferrannini E, Ferri C, Fallahi P. Chemokine (C--X--C motif) ligand (CXCL) 10 in autoimmune diseases. Autoimmun Rev. 2014;13(3):272-80.

9. Lewis SM, Barbara JB, Bates I. Dacie and Lewis Practical Haematology. Dacie Lewis Pract Haematol. 2006;2006.

10. Henrickson KJ. Parainfluenza Viruses. Clin Microbiol Rev [Internet]. 2003;16(2):24264. Available from: https://cmr.asm.org/ content/16/2/242

11. Vainionpaa R, Hyypia T. Biology of parainfluenza viruses. [Review] [117 refs] . Clin Microbiol Rev . 1994;7(2):265-75.

12. El Feghaly RE, McGann L, Bonville CA, Branigan PJ, Suryadevera M, Rosenberg HF, et al. Local production of inflammatory mediators during childhood parainfluenza virus infection. Pediatr
Infect Dis J. 2010;29(4):e26.

13. Weinberg GA, Hall CB, Iwane MK, Poehling KA, Edwards KM, Griffin MR, et al. Parainfluenza Virus Infection of Young Children: Estimates of the Population-Based Burden of Hospitalization. J Pediatr [Internet]. 2009;154(5):694-699.e1. Available from: http://www.sciencedirect.com/ science/article/pii/S0022347608010366

14. Schmidt AC, Schaap-Nutt A, Bartlett EJ, Schomacker H, Boonyaratanakornkit J, Karron RA, et al. Progress in the development of human parainfluenza virus vaccines. Expert Rev Respir Med [Internet]. 2011;5(4):515-26. Available from: https://doi.org/10.1586/ers.11.32

15. Marx A, Marston BJ, Erdman DD, Gary Howard E. J, Plouffe JF, Breiman RF, et al. Parainfluenza Virus Infection Among Adults Hospitalized for Lower Respiratory Tract Infection. Clin Infect Dis [Internet]. 1999;29(1):134-40. Available from: https://doi.org/10.1086/520142

16. Schomacker H, Schaap-Nutt A, Collins PL, Schmidt AC. Pathogenesis of acute respiratory illness caused by human parainfluenza viruses. Curr Opin Virol [Internet]. 2012;2(3):294-9. Available from: http://www.sciencedirect.com/science/article/pii/ S1879625712000284

17. Sumino KC, Walter MJ, Mikols CL, Thompson SA, Gaudreault-Keener M, Arens MQ, et al. Detection of respiratory viruses and the associated chemokine responses in serious acute respiratory illness. Thorax [Internet]. 2010;65(7):639-44. Available from: https://thorax.bmj.com/content/65/7/639

18. Antonelli A, Rotondi M, Fallahi P, Ferrari SM, Paolicchi A, Romagnani $\mathrm{P}$, et al. Increase of CXC chemokine CXCL10 and CC chemokine CCL2 serum levels in normal ageing. Cytokine. 2006;34(1-2):32-8.

19. Hansen S, Baptiste KE, Fjeldborg J, Betancourt A, Horohov DW. A comparison of proinflammatory cytokine mRNA expression in equine bronchoalveolar lavage (BAL) and peripheral blood. Vet Immunol Immunopathol [Internet]. 2014;158(3):238-43. Available from: http://www.sciencedirect.com/science/article/pii/ S0165242714000373

20. Lamparello AJ, Namas RA, Abdul-Malak O, Vodovotz Y, Billiar TR. Young and Aged Blunt Trauma Patients Display Major Differences 
in Circulating Inflammatory Mediator Profiles after Severe Injury. J Am Coll Surg [Internet]. 2019;228(2):148-160.e7. Available from: http:// www.sciencedirect.com/science/article/pii/ S1072751518321562

21. Yan W, Chen C, Chen H. Estrogen Downregulates miR-21 Expression and Induces Inflammatory Infiltration of Macrophages in Polymyositis: Role of CXCL10. Mol Neurobiol [Internet]. 2017
Apr;54(3):1631-41. Available from: https://doi. org/10.1007/s12035-016-9769-6

22. Wilson N, Driss A, Solomon W, DickinsonCopeland C, Salifu H, Jain V, et al. CXCL10 Gene Promoter Polymorphism - $1447 \mathrm{~A}>\mathrm{G}$ Correlates with Plasma CXCL10 Levels and is Associated with Male Susceptibility to Cerebral Malaria. PLoS One [Internet]. 2013;8(12):1-11. Available from: https://doi.org/10.1371/journal.pone.0081329 